\title{
CAPITULO 5 \\ Evaluación de los sistemas de calidad de la información en centros hospitalarios de alta complejidad en la ciudad de Barranquilla
}

\section{Evaluation of information quality systems in high-complexity healthcare centers in the city of Barranquilla}

\author{
BELKIS ESCORCIA ${ }^{1}$ \\ YENNIFER SAAVEDRA BARRAZA ${ }^{2}$ \\ DAVID MARTÍNEZ SIERRA ${ }^{3}$
}

\footnotetext{
* Artículo resultado de la investigación de la tesis de maestría titulada: Sistema de Calidad de Información en la ESE Cari Alta Complejidad, Universidad Simón Bolívar, 2014.

1 Magíster en Auditoría y sistemas de calidad en servicio de salud. bescorcia@unisimonbolivar.edu.co.

2 Magíster en Auditoría y sistemas de calidad en servicio de salud. ysaavedra@unisimonbolivar.edu.co.

3 Docente del programa Maestría en Auditoría y sistemas de calidad en servicio de salud. dmartinez@unisimonbolivar.edu.co.
} 


\title{
RESUMEN
}

En Colombia, la legislación sanitaria tiene como objetivo fundamental la atención oportuna y digna en todo lo referente al sector salud. A pesar de ello, son constantes las quejas que se reciben en los diversos organismos de control y en un gran porcentaje están enfocadas en el manejo de la información.

Por lo anterior, el presente trabajo tendrá como finalidad, analizar los aspectos que coinciden en estos escenarios y establecer qué medidas de manera preventiva y correctiva podrían sugerirse para lograr un mayor desempeño a nivel particular y colectivo en cuanto al manejo de la información en las instituciones de alta complejidad.

Palabras clave: información, paciente, usuario, sistema, salud.

\begin{abstract}
In Colombia, health legislation has as its fundamental objective timely and dignified attention in all matters relating to the health sector. In spite of this, the complaints that are received in the various control organisms are constant and in a great percentage they are focused on the handling of the information.
\end{abstract}

Therefore, the present work will aim to analyze the aspects that coincide in these scenarios and to establish what preventive and corrective measures could be suggested to achieve a greater performance at a particular and collective level, in terms of the management of information, in Institutions of high complexity.

Key words: information, patient, user, system, health. 


\section{INTRODUCCIÓN}

Actualmente los sistemas de información y comunicaciones han llegado a todas las instancias de la sociedad, y la salud no es la excepción. Toda la información derivada de un proceso de atención se encuentra ahora inserto en un complejo compendio, donde debe primar la organización y control, para poder conseguir la gestión y satisfacción oportuna con cada uno de los usuarios en todos los niveles.

Este nuevo escenario ha traído consigo muchas ventajas, pero también involucra algunos factores que deben ser atendidos para lograr el objetivo propuesto. Uno de esos aspectos está relacionado con la calidad de la información, la cual se puede ver afectada por agentes internos y externos de cada organización prestadora de servicios de salud.

Son muchas las reclamaciones que los usuarios interponen ante las entidades regulatorias, y en su gran mayoría hacen referencia a las demoras injustificadas, retardos en las respuestas, falta de controles para evitar la sustracción y pérdida de la información general que se mueve en el sistema. Toda esta realidad requiere procesos de intervención que promuevan las estrategias necesarias y que, siguiendo los lineamientos legales, contribuya con la gestión oportuna y de calidad en las entidades de salud.

En la investigación definida, mediante un análisis DOFA, se analizará esta problemática con la finalidad de examinar tanto fortalezas como debilidades, y a partir de la identificación de amenazas y oportunidades, entregar reflexiones de acompañamiento.

El objetivo central será evaluar y valorar los sistemas de información para lograr que estos faciliten el acceso a los datos generados de manera diversa y dispersa, por distintas organizaciones con el propósito de disponer 
en tiempo y forma, de un conocimiento confiable y estructurado sobre un ámbito de la vida tan primordial como lo es la salud (Organización Panamericana de la Salud, 2007).

\section{SISTEMAS DE INFORMACIÓN EN INSTITUCIONES PRESTADORAS DE SERVICIOS DE SALUD}

Las Guías para prácticas clínicas de trastornos hipertensivos gestacionales son compendios emanados por entidades de carácter gubernamental en la rama de salud o entidades educativas en sus áreas de investigación científica y desarrollo, que contienen observaciones, indicaciones y/o recomendaciones, que llevadas de manera metódica, contienen los saberes y competencias necesarias para que los actores principales de las entidades de salud (médicos, enfermeras, paramédicos) apliquen de una manera adecuada y eficiente, procedimientos de atención a pacientes femeninas en estado de embarazo garantizando así, excelentes resultados y además, logrando unificar en todas las E.S.E. y sus representantes de servicio estos procedimientos en un ámbito regional (Arrieta, Blanquicet, Borrero \& Muñoz, 2015) (Rodríguez-Dennen et al, 2011).

La aplicación de estas Guías en las entidades de salud tiene ciertas ventajas: los autores son profesionales en el ramo, tienen una amplia trayectoria académica y clínica en el tema que se está tratando (Ortega, Hernández, Mariano \& Herrera 2015). Las Guías también logran sintetizar un gran volumen de información en formatos sencillos, de fácil comprensión y utilización, facilitan la toma de decisiones en el personal involucrado en los casos que hacen referencia al tema médico en mención, el uso de las guías propende por lograr equilibrios entre teoría y práctica, entre actores activos y pasivos de estos escenarios de la salud, y entre experiencias de una u otra persona; además, se pueden adaptar Guías del ámbito nacional para 
aplicarlas en un entorno local (Kovanci \& Gregg, 2010; Salas Paredes et al, 2004; Plazzotta, Luna, \& González Bernaldo de Quirós, F, 2015).

Estas Guías deben ser diseñadas de manera que su contenido sea claro, lógico, conciso, fácil de seguir, no deben presentar desviaciones o tergiversar la información que en ella se contiene, su contenido debe ser comprensible para que cualquier profesional lo pueda entender, debe ser flexible para que pueda aplicarse a cualquier paciente dentro de la diversidad que pueda presentarse, y sus autores deben ser profesionales de diferentes especialidades pero que sean relacionadas con el tema en cuestión (Thompson, Williams \& Miller, 2009).

\section{METODOLOGÍA}

Para poder obtener resultados confiables de un análisis que estime el objetivo principal de este tema, que es saber si los profesionales del E.S.E. Niño Jesús de Barranquilla, conocen la Guía de prácticas clínicas para trastornos hipertensivos gestacionales, se ha determinado que el método a utilizar para este estudio ha de ser una investigación de tipo cuantitativo, ya que lo que se busca analizar a un grupo de individuos que se dividen en forma aleatoria en subgrupos y se analizan con respecto a un factor de medida en el E.S.E. Hospital Niño Jesús de la ciudad de Barranquilla, en el departamento del Atlántico.

Para la determinación del estudio se tomó una muestra de 11 individuos y se tomó una herramienta de captura de datos, llamada el método estadístico Kuder Richarson que usa respuestas dicotómicas para obtener el dato deseado.

\section{RESULTADOS}

Una vez cumplida la etapa de recopilación y análisis de la información, se 
procede a reunir los datos obtenidos y exponer los factores inherentes al proceso de investigación.

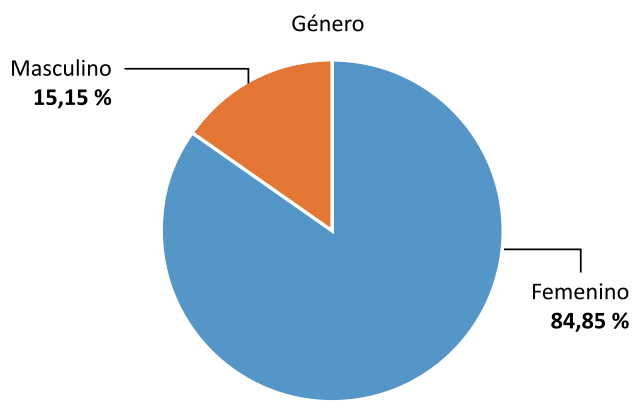

Figura 1

Caracterización de la Población

El resultado de este primer acercamiento, nos muestra que el 84,85 \% de la población objeto de análisis de estudio, pertenecen al género masculino, mientras el 15,15\% restante, pertenecen al género femenino.

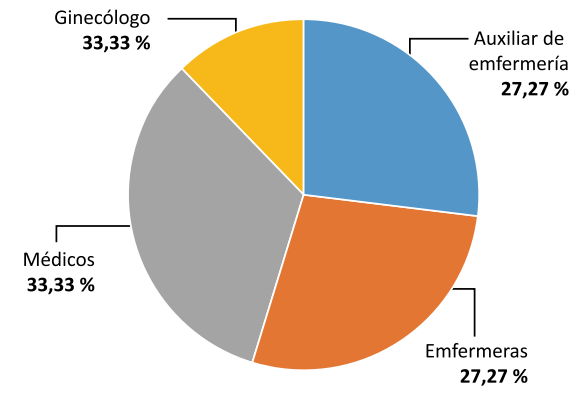

Figura 2

Perfil profesional de la Población en estudio

Según los resultados del gráfico, se indica que el 33,33 \% de los entrevistados son médicos de profesión, un 27,27 \% son enfermeras, y otro $27,27 \%$ son auxiliares de enfermería y el 12,12 \% restante, son médicos especialistas en ginecología. 
Evaluación de los sistemas de calidad de la información en centros hospitalarios de alta complejidad en la ciudad de Barranquilla

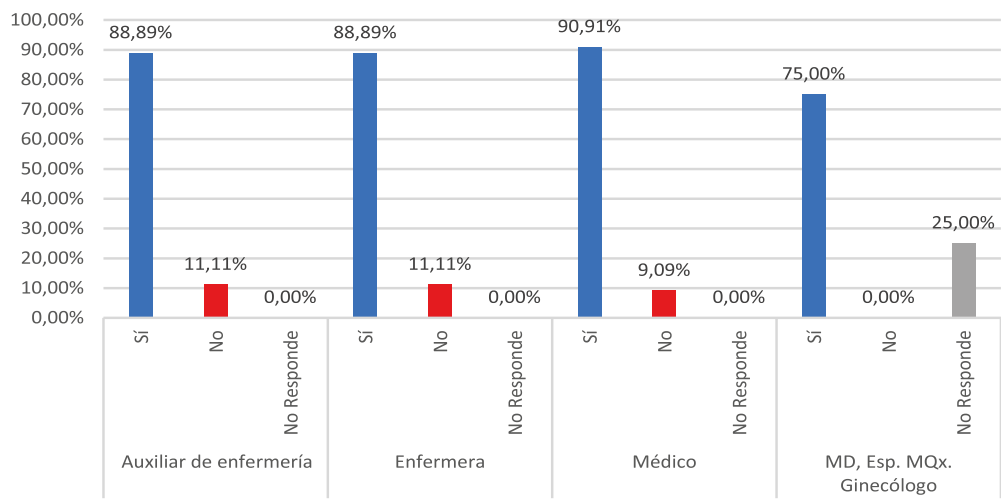

Figura 3

Conocimiento de existencia y aplicación Guías de Prácticas Clínicas

Según los resultados reflejados en este gráfico, aproximadamente un $85 \%$ en promedio de la población en estudio (enfermeras, auxiliares, médicos y ginecólogos) sí tienen conocimiento de la existencia de las Guías de Prácticas Clínicas para trastornos hipertensivos gestacionales, mientras que un $10 \%$ aproximadamente, dice no tener conocimiento sobre estas y un $6,25 \%$ promedio, no responde al interrogante.

Según la ilustración, al momento de tomar decisiones la prioridad la tienen los médicos con un $27,27 \%$ y lo hacen consultando con colegas y otros profesionales, el segundo lugar lo tienen los médicos especialistas y ginecólogos con un 25 \% y lo hacen atendiendo la Guía de Prácticas Clínicas, le siguen con otro $25 \%$ los médicos especialistas en ginecología, basándose en la experiencia, y compartiendo un 18,18 \% los médicos, toman decisiones basados en sus conocimientos. Otro 18,18 \% de profesionales de la salud, pertenecientes a esta muestra lo hacen ayudándose con libros, y por último, un 18,18 \% de médicos lo hacen con la ayuda de la Guía Práctica Clínica. 
Investigaciones y casos del sector salud de la región Caribe

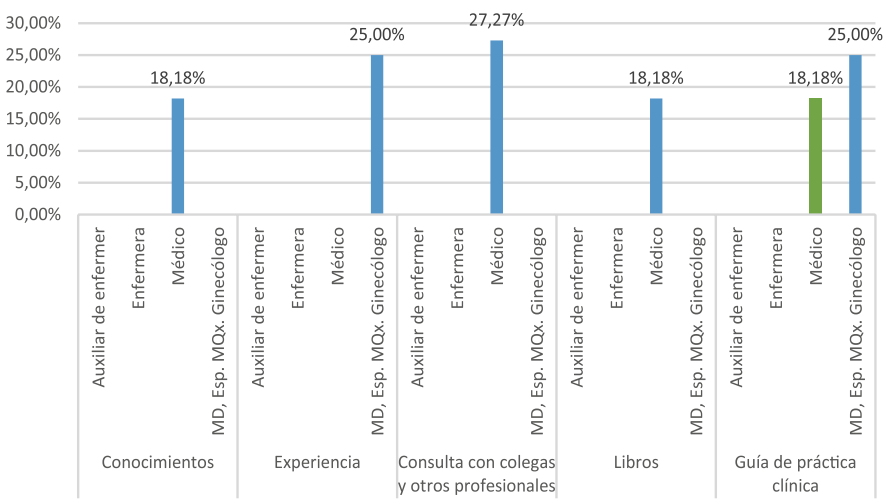

Figura 4

Criterio para toma de decisiones

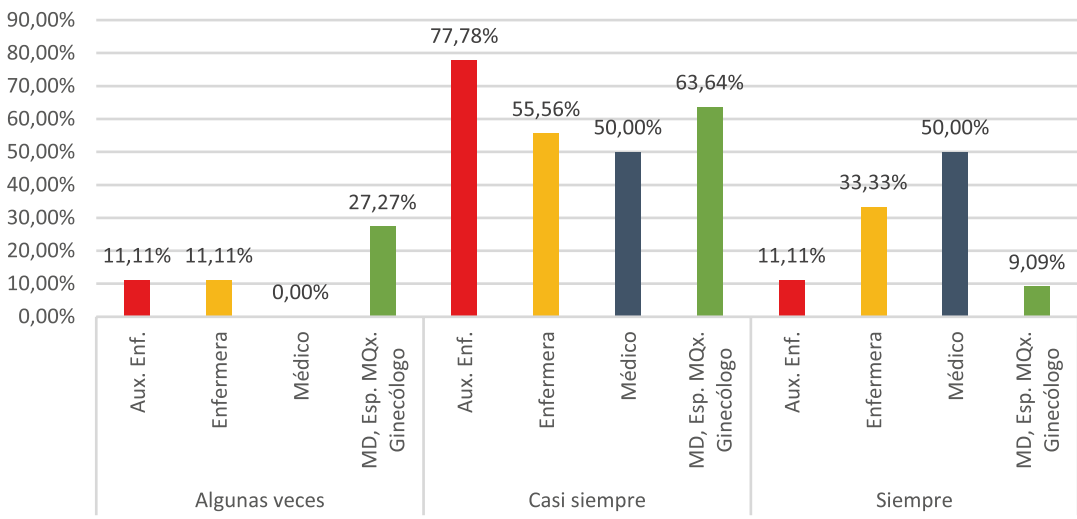

Figura 5

Niveles de adherencia a la Guía práctica de la Clínica

De acuerdo a la encuesta, los especialistas, médicos, enfermeras y auxiliares de enfermería muestran tener con un 61,75 \% "Casi siempre" un buen nivel de adherencia con la Guía práctica clínica, seguido de un 25,88 \% que muestra que "siempre" se ciñen a la guía práctica clínica, y finalmente un 12,37 \% “algunas veces” siguen la guía práctica clínica. 
Evaluación de los sistemas de calidad de la información en centros hospitalarios de alta complejidad en la ciudad de Barranquilla

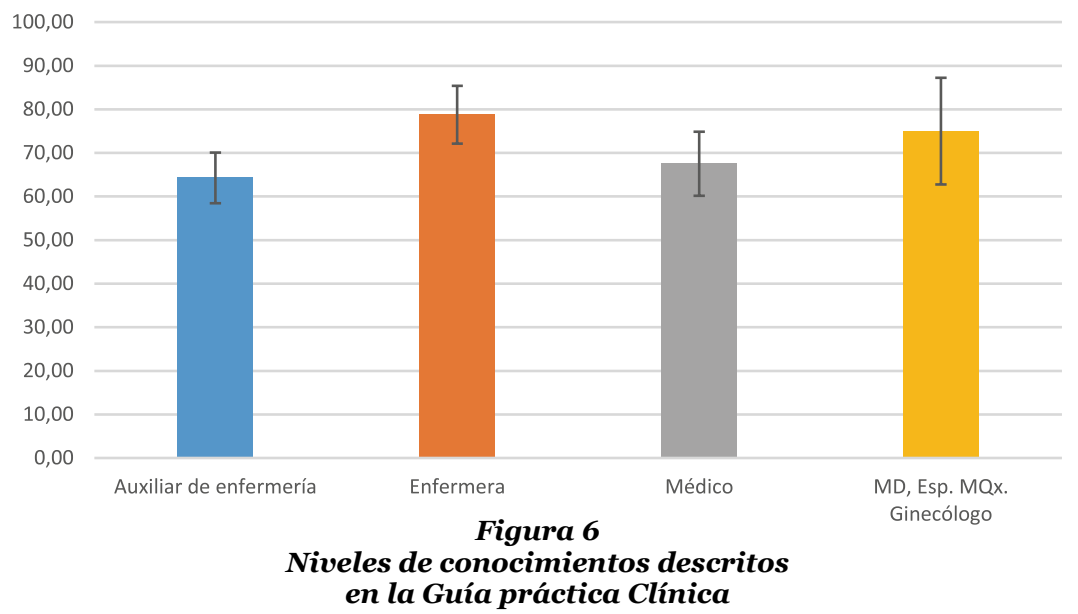

Lo que permite observar este gráfico, es que los niveles de conocimientos de los procedimientos descritos en la Guía práctica clínica, se manejan en su mayoría en un 79 \% por las enfermeras, seguidos de un $74 \%$ por los médicos especialistas en ginecología, el tercer lugar lo tienen los médicos con un $68 \%$, y por último las auxiliares de enfermería con un $64 \%$.

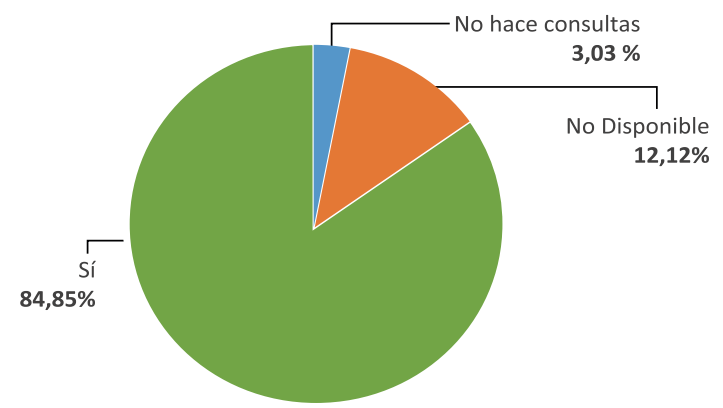

Figura 7

Consultas a la guía de prácticas clínicas por el personal de la E.S.E.

Como se evidencia, un 84,85\% dice que, Sí hace consultas en la guía para prácticas clínicas, seguido de un 12,12 \% que dicen No tener disponible la guía para prácticas clínicas y un 3,03 \% dice que no hace consultas a este compendio. 


\section{CONCLUSIONES}

En el ejercicio de la exploración de la adherencia de los actores del ámbito de salud en la ciudad de Barranquilla, mediante el análisis realizado en la muestra seleccionada, se pudo evidenciar el estado de la categorización y calificación, del estado del conocimiento sobre la adherencia a las Guías prácticas clínicas en las ESE del Estado.

Los resultados finales permiten enunciar que el estado de adherencia actual se encuentra en un nivel alto que se proyecta en auge con referencia a todo el personal involucrado en la atención de la madre gestante con trastornos hipertensivos. Solo un 10 \% de la población indagada desconoce la existencia y alcance total de las guías de prácticas médicas, y un porcentaje cercano al $6 \%$ no supo responder a este interrogante. Como lo ha expuesto Bertoglia et al (2010), la cultura y dinamización del conocimiento en el campo médico es una labor constante que debe alimentarse sin cansancio. Lo anterior cobra pertinencia en el caso de estudio, ya que al promover el aprendizaje constante por parte de todos los actores médicos, los resultados pueden ser tanto óptimos como oportunos.

Otro aspecto a resaltar es la de la consulta a la Guía; 84 \% viene realizando retroalimentación con el compendio, lo que brinda garantía y tranquilidad al interior de las IPS, ya que como lo ha expresado Gaillard et al (2011), en la medida que las herramientas generadas por los entes de investigación gubernamental o no gubernamental sean consultadas, hay más posibilidades de acierto en cada uno de los diferentes casos que se pueden presentar, especialmente, en sucesos de urgencia con madres gestantes.

Los hallazgos en términos generales dejan un positivo balance en cuanto a la difusión y conocimiento de la Guía de prácticas clínicas, sin embargo, es conveniente continuar con los programas de promoción y lograr la captura de atención de los profesionales, ya que aunque la Guía está a disposición en momentos cruciales, las decisiones se vienen tomando con base en con- 
ceptos de colegas, consulta con otras fuentes o revisiones de casos anteriores. Finalmente, se puede concluir que la importancia de los programas de promoción al interior del sector salud, especialmente las ESE del Estado, son cruciales, no solo es necesario contar con el material de consulta, sino además, estimular que todas las herramientas e instrumentos diseñados para su aplicación sean tomados en cuenta, entre otros aspectos, porque una adecuada decisión puede salvar vidas e impactar de manera directa en las estadísticas negativas que quieren erradicarse en este campo.

\section{REFERENCIAS BIBLIOGRÁFICAS}

Arrieta, N., Blanquiceth, R., Borrero, E. \& Muños, V. (2015). Impacto del Programa de Hipertensión Arterial en un Centro de Salud de Soledad, Atlántico (Col). Universidad Simón Bolívar. Revista Ciencia e Innovación en Salud, 3(1), 27-32.

Bertoglia P, Rivas A, Navarrete P, Castro M L, Acurio J, Escudero C. (2010).

Resultados clínicos y perinatales de los embarazos con hipertensión arterial en un hospital de referencia de la VIII región de Chile. Rev Chil Obstet Ginecol, 75, 162-171.

Gaillard R, Bakker R, Willemsen P, Hofman A, Steegers E, Jaddoe WV. (2011). Blood pressure tracking during pregnancy and the risk of gestational hypertensive disorders: The Generation R Study. Eur Heart J., 32, 3088-3097.

Kovanci E, Gregg A. (2010). Blood Pressure Regulation Across Pregnancy: Evidence of a Paradigm Shift in Gene Expression. Hypertens Pregnancy, 29(2), 236-247.

Landázuri P, Restrepo B, Trejos J. (2006). Perfil lipídico por trimestres de gestaciones en una población de mujeres colombianas. Rev Colomb Obstet Ginecol, 57, 256-263.

LilDommar, Suárez C, Rojas G, Marcano M, Nuccio J. (2009). Hipertension Arterial Inducida por embarazo: Factores de riesgo asociados. $\mathrm{Sa}$ ber, 21, 34-39. 
MacDonald-Wallis C, Lawlor, Debbie A, Fraser A, May M, Nelson Scott $\mathrm{M}$, et al. (2012). Blood Pressure Change in Normotensive, Gestational Hypertensive, Preeclamptic, and Essential Hypertensive Pregnancies. Hypertension, 59, 1241-1248.

Ortega, J., Hernández, H., Mariano, H. \& Herrera, A. (2015). Calidad Estratégica en los Servicios de Salud. Centro de investigación y proyectos (CINPRO) Corporación Universitaria Latinoamericana.

Plazzotta, F., Luna, D., \& González Bernaldo de Quirós, F (2015). Sistemas de información en salud: integrando datos clínicos en diferentes escenarios y usuarios. Rev. perú. med. exp. y Salud Pública, 32(2), 343-351. Disponible en: <http://www.scielo.org.pe/scielo.php?script=sci_arttex\&pid=S1726-46342015000200020\&Ing=es\&nmr=iso $>$.

Rodríguez-Dennen F, Martínez Ocaña J, KawaKarasik S, Villanueva Egan L, Reyes Paredes N, Flisser A, et al. (2011). Comparison of hemodynamic, biochemical and hematological parameters of healthy pregnant women in the third trimester of pregnancy and the active labor phase. BMC Pregnancy Childbirth, 11, 11-33.

Romero-Gutiérrez G, González B. (2011). Persistencia de hipertensión en mujeres con preeclampsia. Ginecol Obstet Mex, 79, 601-606.

Salas Paredes AJ, Velázquez Maldonado E, Villarroel V, Arata Bellabarba G. (2004). Relación entre la concentración de leptina, insulina, norepinefrina y presión arterial en embarazadas sanas. Rev Venez Endocrinol Metab, 2, 10-14.

Thompson M, Williams M, Miller R. (2009). Modelling the association of blood pressure during pregnancy with gestational age and body mass index. Paediatr Perinat Epidemiol, 23, 254-263.

Cómo citar este capítulo:

Escorcia, B., Saavedra Barraza, Y., \& Martínez Sierra, M. S. (2017). Evaluación de los sistemas de calidad de la información en centros hospitalarios de alta complejidad en la ciudad de Barranquilla. En J. Rodríguez López, M. Gómez Barbosa, D. Martínez Sierra, H. Hernández Palma, C. Sierra García, C. Beleño Agudelo, . . . J. Bermejo Urzola, Investigaciones y casos del sector salud de la región Caribe (pp.73-84). Barranquilla: Ediciones Universidad
Simón Bolívar. 